\title{
Forest chimpanzees (Pan troglodytes verus) remember the location of numerous fruit trees
}

\author{
Emmanuelle Normand · Simone Dagui Ban • \\ Christophe Boesch
}

Received: 12 November 2008 / Revised: 11 May 2009 / Accepted: 12 May 2009 / Published online: 31 May 2009

(C) The Author(s) 2009. This article is published with open access at Springerlink.com

\begin{abstract}
It is assumed that spatial memory contributes crucially to animal cognition since animals' habitats entail a large number of dispersed and unpredictable food sources. Spatial memory has been investigated under controlled conditions, with different species showing and different conditions leading to varying performance levels. However, the number of food sources investigated is very low compared to what exists under natural conditions, where food resources are so abundant that it is difficult to precisely identify what is available. By using a detailed botanical map containing over 12,499 trees known to be used by the Taï chimpanzees, we created virtual maps of all productive fruit trees to simulate potential strategies used by wild chimpanzees to reach resources without spatial memory. First, we simulated different assumptions concerning the chimpanzees' preference for a particular tree species, and, second, we varied the detection field to control for the possible use of smell to detect fruiting trees. For all
\end{abstract}

E. Normand $(\varangle) \cdot$ C. Boesch

Department of Primatology,

Max Planck Institute for Evolutionary Anthropology,

Deutscher Platz 6, 04103 Leipzig, Germany

e-mail: normand@eva.mpg.de

E. Normand

School of Cognitive Sciences,

Victor Segalen Bordeaux 2 University, Bordeaux, France

S. D. Ban

Université de Cocody, Abidjan, Côte d'Ivoire

S. D. Ban

Centre Suisse de Recherche Scientifique, Abidjan, Côte d'Ivoire these assumptions, we compared simulated distance travelled, frequencies of trees visited, and revisit rates with what we actually observed in wild chimpanzees. Our results show that chimpanzees visit rare tree species more frequently, travel shorter distances to reach them, and revisit the same trees more often than if they had no spatial memory. In addition, we demonstrate that chimpanzees travel longer distances to reach resources where they will eat for longer periods of time, and revisit resources more frequently where they ate for a long period of time during their first visit. Therefore, this study shows that forest chimpanzees possess a precise spatial memory which allows them to remember the location of numerous resources and use this information to select the most attractive resources.

Keywords Spatial memory - Navigation simulation . Chimpanzee $\cdot$ Pan troglodytes verus

\section{Introduction}

Spatial memory is a central topic in the study of animal cognition because animals' habitats are generally large, with food sources often being dispersed and unpredictable. Studies on the subject range from testing for the existence of spatial memory in different species to deciphering the precise ability used to select specific resources (e.g., the closest or the most productive; Garber 1988; Janson 1998), to testing for the ability to plan for the future (e.g., very distant resources or entire day long journeys; Noser and Byrne 2007; Raby et al. 2007). One critical aspect in the study of spatial memory in animals is to account for an individuals' prior spatial knowledge of the area in which they live. This was initially tested in laboratory animals using experiments 
aimed at measuring the ability to revisit a place in the most efficient way (Morris' swimming pool, Morris 1981), or to avoid places that had already been visited for food or that are known to be empty (radial maze, Beatty and Shavalia 1980). For revisits to a particular resource, animals were tested on their ability to learn and remember how to return directly or more efficiently to a particular place (Menzel 1973).

Nevertheless, when spatial memory is tested under controlled conditions, animals have to memorize a small number of food places (e.g. eight resources in the eight-arm radial maze, Beatty and Shavalia 1980; or 136 bowls for sheep on $80 \times 80$ or $160 \times 160 \mathrm{~m}$ area, Dumont and Petit 1998). Similarly, while testing for the ability to uncover hidden food places, Menzel (1973) showed that chimpanzees were able to memorize 17 food places out of the 18 shown. In addition, there is some evidence that tests of spatial ability in captive animals result in different findings than such tests in their wild counterparts. For example, Menzel and Beck (2000) found that captive-reared golden tamarins that had been reintroduced to a reserve were deficient in travel patterns compared to wild tamarins since they appeared to occasionally get lost less than $50 \mathrm{~m}$ from their nest box and often could not work out a spatial route to return to it, even when it was in their direct view. This apparent incompetence is in contrast with Garber and Paciulli's (1997) findings that showed that ecologically similar wild tamarins have a well-developed spatial memory, even for temporary resources found across hundreds of meters. Therefore, on the one hand, experiments provide us with knowledge on what animals remember from prior experience by controlling the number of resources and their locations. However, on the other hand, studies on animals' spatial memory in their natural habitats are necessary to understand what abilities have been developed by wild animals in complex and rich habitats, comprised of a high number of resources that are spread over very large areas. Wild animals must develop precise spatial abilities and integrate the location of a large number of resources with time to be able to travel efficiently. Finding the most productive resources in the most efficient way is essential for any animal species, and animals seem to be able to remember diverse information about the location and type of food (scrub jays: Clayton and Dickinson 1998; domestic pigs: Held et al. 2005). Recently, there has been a growing interest in spatial memory in primates; for example, studies have looked at how efficient and useful this memory is for remembering where (Janson 1998; Janson 2007), when (Janmaat et al. 2006), or what (Garber 1989; Janson 2007) resources produce. A recent study demonstrated the existence of a precise Euclidean map in wild chimpanzees (Normand and Boesch 2009), showing that they navigate between resources efficiently and precisely. Therefore, primates and especially chimpanzees seem to have developed outstanding abilities to navigate in their complex natural environment. However the question of how much they know about their complex environment remained difficult to assess as it is difficult and perhaps even impossible for researchers to precisely know the fructification state of all resources in a large territory in the wild, as, for example, is the case with a large $20 \mathrm{~km}^{2}$ chimpanzee territory.

Nevertheless, in the past, the availability of resources has been evaluated using a variety of approaches. For example, some studies have considered all resources that were recorded during the previous years (Garber 1989), and others have used these resources as the basis for phenology studies, in order to gain more precise information on resource availability (Cunningham and Janson 2007). In other studies, the focus was limited to one or two particular food species for which a certain number of locations were listed during the study period (Janmaat et al. 2006). Alternatively, some experiments have been carried out in the wild using feeding platforms during periods of low natural food availability, as a way to control for the availability of resources (Janson 1998). Using this last technique, monkeys have been shown to remember the location of a few platforms and travel efficiently to the next closest one (Janson 1998). However, the tropical rainforests in which the majority of primates live are characterized by a much higher level of tree species diversity and by unpredictable food productivity. With such rich food productivity in an environment with low visibility, it is possible that there is either so much food available that navigating in any direction would eventually lead to a resource (Janson and Byrne 2007), or that spatial memory is needed because the chance of finding a productive resource at random would be extremely time consuming and energy demanding. Simulations of navigation that do not rely on spatial memory have been used to test whether primates find resources by chance or by smell (Janson 1998; Garber and Hannon 1993). When compared to observations of how animals actually navigate, results demonstrated that primates were more efficient in travelling to food resources than they would be simply by chance.

To test whether wild chimpanzees use a precise and large spatial memory, we accurately mapped all trees of the 17 important food tree species eaten by chimpanzees in the Taï National Park, Côte d'Ivoire $(N=12,499$ trees in a $15 \mathrm{~km}^{2}$ area). Using this map, we first tested if chimpanzees have a spatial memory. To test for the possibility that chimpanzees might be navigating in the forest without using spatial memory, we ran simulations of navigation, integrating all productive trees of the species eaten by the chimpanzees during a given month. By comparing the simulation output with natural observations, we tested whether it is possible for chimpanzees to find rare resources by chance, 
and how efficient they would be when navigating randomly through forest resources. Second, we searched for the criteria chimpanzees use to select the trees. We predicted that chimpanzees memorize the locations of the available resources and revisit the same resource more often and more efficiently than would be expected by chance. Furthermore, if they can remember the location of many trees over time, then it would be beneficial for them to choose to visit the trees that have a more abundant fruit production.

\section{Method}

Study site and subjects

The research was carried out in the Taï National Park, Côte d'Ivoire, on chimpanzees in the South Group (Boesch and Boesch-Achermann 2000; Boesch et al. 2006b, 2008). The South Group has been fully habituated since 1995 and the individuals are followed on a regular basis. The present study began in 2004 with the creation of a detailed map of the resources in the territory. Data collection on the chimpanzees' navigation was carried out between February 2005 and July 2007. The chimpanzee community was composed of 16 adult chimpanzees (11 females and 5 males). Of these individuals, 15 were followed for 375 days. The remaining individual, an adult female, was not followed as she was not completely habituated to humans. For 3 months during the study period, two females were followed for 28 consecutive days (from 5 January to 1 February 2007, and from 19 February to 18 March 2007) to study how chimpanzees revisit the same trees. At other times, different group members were followed whenever it was possible and for more than 1 day when they left the group by themselves, in order to understand individual knowledge and to study social influence on navigation.

\section{Behavioural data collection}

Each day, a different target individual was chosen for the data collection of that given day. The individual was followed from the morning, when he was leaving his sleeping nest (around 6:30 a.m.), until the evening, when the individual built a new nest (around 6:00 p.m.). The location of the target was recorded with a GPS Garmin 60 in 2005, and a GPS Garmin 60cx from 2006 on. The GPS recorded the location of the observer following the target chimpanzees, who had to be at least $7 \mathrm{~m}$ away (this distance rule was implemented in an effort to avoid disease transmission from humans to chimpanzees). The coordinates were transferred in UTM units (universal transverse mercator), which provide data in meters. We recorded activities and position, including when the chimpanzees stopped travelling. At each of these points, we recorded the names of all individuals observed. This allowed us to determine the number of individuals (mean party size $=4.35$; mean female party size $=2.85$ mean; mean male party size $=1.5$ ) and the female ratio (mean $=0.69)$, two variables used in this study to control any variation of navigation patterns for social factors (Normand and Boesch 2009). The duration of each eating bout was recorded, as well as the amount of time spent resting. Feeding time at one tree was calculated as the sum of the individual feeding bouts.

\section{Botanical map}

During the study, the South Group territory used by the chimpanzees had a total area of $25 \mathrm{~km}^{2}$ and all individual trees of 17 known tree species that the chimpanzees fed on were mapped with a GPS in a section that was approximately $60 \%$ of this territory. We selected these 17 tree species, which are important food sources for the chimpanzees and have varying densities and distributions in the forest, so that we could better understand the orientation of the chimpanzees while foraging. Of the trees eaten by chimpanzees, $69.3 \%$ were located in the mapped area. Tree positions were recorded with a GPS following virtual transects that we created with Mapsource. Each transect was separated by a distance of $15 \mathrm{~m}$ so that no trees would be missed. A map of all trees, with different layers representing different tree species, was created with ArcView. In this way, we identified and recorded 12,499 trees from March 2004 to November 2007, with the help of two trained field assistants.

Simulation of navigation without spatial memory

To be able to evaluate the theoretical frequency of visits as well as the distance travelled without spatial memory, we needed a map that would not only include the exact positions of all trees of each of the different species eaten by the chimpanzees, but that would also give us an indication about the fruit productivity of each tree. As we recorded 12,499 trees, it was not possible to evaluate each tree every month for fruit production. Therefore, for the simulation, we produced virtual maps of the productive trees for each month. The program extracted a randomly selected proportion of trees of the target species. The tree production value entered for each species within any given month was based on our long-term phenological data for the South Group territory (Boesch et al. 2006a). Next, we let the simulation produce 100 virtual maps at random (for an example, see Fig. 1) and ran each of them 100 times for each of the different travel conditions. For example, to test how often chimpanzees would find fruiting Parinari excelsa trees without using spatial memory in January 2007, we produced virtual maps with the same proportion of Parinari 
Fig. 1 Example of a virtual map produced for simulating movements that considers more than one species, showing the position of the productive trees in January 2007. The productive trees had been chosen at random with a program in Visual Basic (see text for more explanations). The simulations produced 100 different virtual maps, where productive trees were selected at random. For each map, the program runs a virtual search by a chimpanzee from a different initial point to different selected trees 100 times

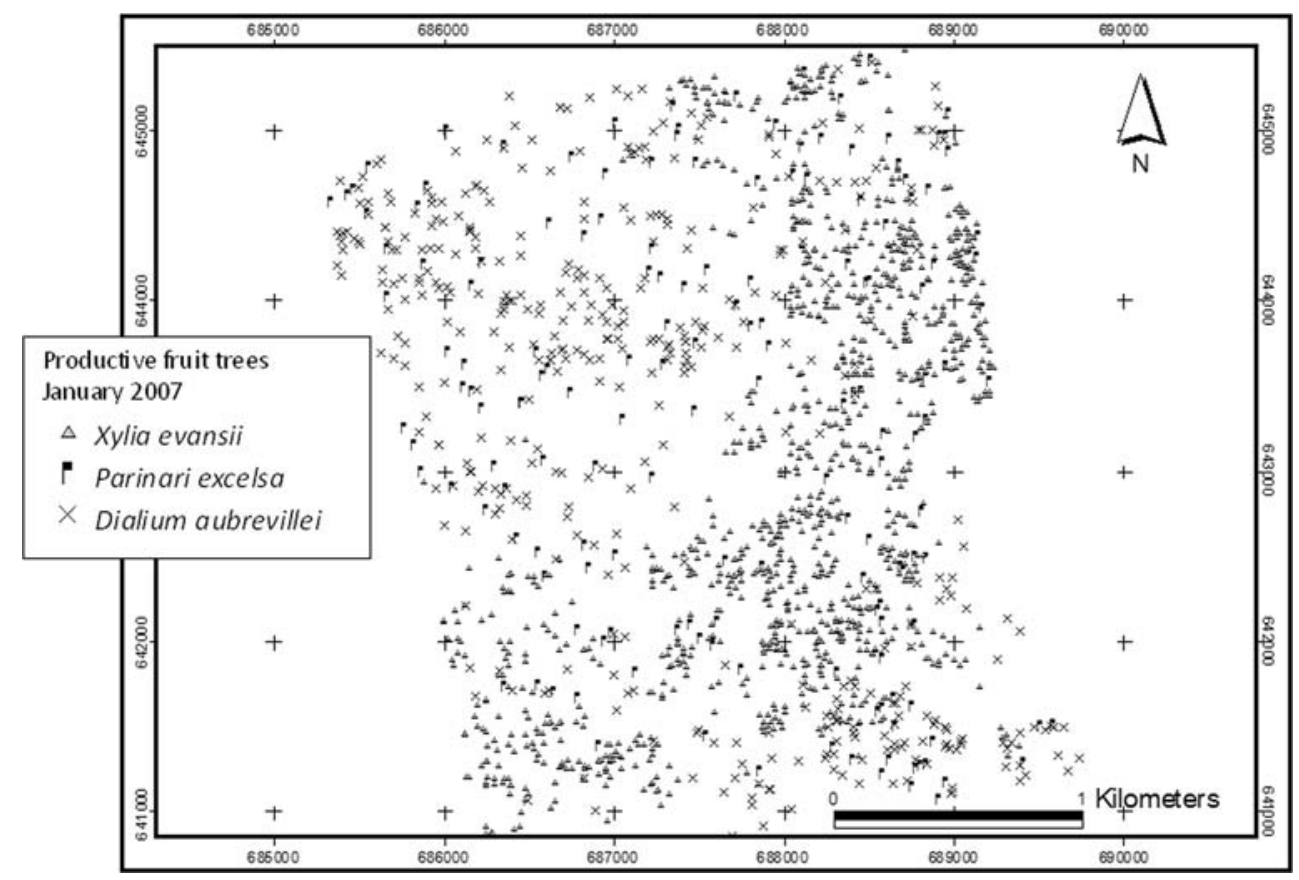

trees that we knew were producing fruit from our phenology survey (28\% of the Parinari trees were carrying fruit that month) (Fig. 1). During one simulation (see below), we would randomly produce 100 new virtual maps while keeping the production rate constant for the tree species tested and have the simulation start from a new predetermined point each time.

The initial position in the simulation is a tree that is randomly selected in the virtual map and the first travelling direction is also randomly selected. The initial direction is kept until a tree is found, thereby reproducing the linearity of the movement patterns observed in Taï chimpanzees between resources (Normand and Boesch 2009). Once the run starts, the first tree encountered becomes the new position. The next direction is determined using a general deviation rate estimated from the observed deviation a chimpanzee follows between the direction adopted to reach a resource and the direction selected when he leaves the resource. As chimpanzees rarely backtrack, this parameter increases the realism of the simulations. At the end of each trial, the program determines the visit and revisit rates for each tree species and the mean distance travelled.

Since we cannot know if a chimpanzee leaves a tree with a precise idea about which tree species he is going to next, we simulated three alternatives. In the first one, the "no preference" simulation, the target chimpanzee does not select any particular tree species but, instead, goes to the first tree he detects. For this simulation, we only included tree species that represented more than $10 \%$ of the total number of trees they visited during the study month. In this way, we obtained the frequency and the distance of each visit to a particular tree species as well as the distance to revisit them under the "no spatial memory" and the "no preference" models. In the second simulation, the "preference" simulation, the target chimpanzee decides which tree species to visit before leaving the previous one and this is entered into the simulation so that it will continue to run until the appropriate tree species is encountered. Table 1 presents the data from the 4 months used in the analysis, in which we had enough observations for all trees of the species that were consumed within 1 month. As can be seen, tree production, as well as the number of trees in production for the same species, varies greatly between the monthly periods. In addition, the number of trees in production is very different for the various tree species eaten by the chimpanzees. To be safe, we tested a third, mixed simulation, the "varying preference" model, in which the target chimpanzee starts with the preference rules but, after a certain distance, switches to the non-preference rules. Here, we simulated the switch after 100 or $200 \mathrm{~m}$. Moreover, for all alternatives, if more than one tree is in the detection field, the target chimpanzees will visit the preferred species.

Finally, at only $30 \mathrm{~m}$ (Boesch and Boesch-Achermann 2000), the visibility in the Taï forest is low. Preliminary results from a study that began in December 2008 confirm that, for 40 trees inspected (Parinari, Xylia, Dialium), the visual detection field to detect a tree trunk was an average of $36 \mathrm{~m}(\mathrm{~min}=18 \mathrm{~m}$ and $\max =50 \mathrm{~m})$ and an average of $31 \mathrm{~m}$ for the tree crown $(\min =14 \mathrm{~m}$ and $\max =50 \mathrm{~m})(\mathrm{Ban}$ D.S. and Janmaat K., personal communication). Thus, we used a detection field of $30 \mathrm{~m}$ to study navigation without spatial memory using visual cues, and then two other 
Table 1 Chimpanzee's tree species preferences and their productivity characteristics for four different months in the Taï forest

The third column shows the number of trees visited by chimpanzees and the fourth how frequently they ate from that species. The last two columns present the proportion of productive trees for each species and the related number of trees available in the territory

\begin{tabular}{llcllc}
\hline Period & Species & $\begin{array}{l}\text { Observation } \\
\text { (cases) }\end{array}$ & $\begin{array}{l}\text { Visit } \\
\text { frequency }\end{array}$ & $\begin{array}{l}\text { Proportion of } \\
\text { trees producing }\end{array}$ & $\begin{array}{l}\text { Number of } \\
\text { trees producing }\end{array}$ \\
\hline March 2005 & Pouteria & 29 & 0.420 & 0.750 & 93 \\
& Dialium & 18 & 0.261 & 0.911 & 1164 \\
January 2007 & Xylia & 22 & 0.319 & 0.416 & 1143 \\
& Parinari & 100 & 0.546 & 0.286 & 163 \\
& Dialium & 45 & 0.246 & 0.333 & 425 \\
February 2007 & Xylia & 38 & 0.208 & 0.417 & 1146 \\
& Parinari & 127 & 0.585 & 0.357 & 203 \\
& Chrysophyllum & 52 & 0.240 & 0.750 & 1347 \\
March 2007 & Xyl a & 38 & 0.175 & 0.500 & 1374 \\
& Parinari & 152 & 0.670 & 0.286 & 163 \\
& Xylia & 75 & 0.330 & 0.083 & 228 \\
\hline
\end{tabular}

detection fields, 60 and $100 \mathrm{~m}$, to account for possible alternative detection means such as smell, the sound of fruit falling, or vocalisations. Moreover, this allowed us to study whether our simulations make sense in relation to the detection field.

To calculate the frequency of tree visits, we divided the number of trees of each species that the chimpanzees visited by the number of trees for all considered species that were visited during the period. Finally, for the distance travelled, we considered the distances measured by the GPS when following chimpanzees between visited trees shown in Table 1. Furthermore, we considered only species for which there were more than ten visits observed during a given month. We compared the simulated distance travelled, revisit rates, and visit frequency determined for each species with the observation patterns of the same species.

Determining fruiting pattern of tree productivity

Because we were unable to evaluate the fruit production state of each of the 12,499 mapped trees, we assumed that their production pattern could be reliably predicted from the monthly phenology data that included a subset of 224 individuals of those mapped trees. We used the trees included in the phenology data to control for possible spatial clusters in the fruiting pattern, as the likelihood of finding them without spatial memory would be higher for clustered productivity. To determine the spatial distribution patterns of productive trees, we ran spatial autocorrelation analyses on the phenology data using the Moran's I index from ArcGis. This index evaluates whether the pattern expressed is clustered, dispersed, or random. A $z$-score outside the \pm 1.65 interval indicates statistical significance. If significant, a Moran's $I$ value near +1.0 indicates clustering, while a value near -1.0 indicates dispersion. For the 3 months considered (March 2005, January 2007 and February 2007), we found the following Moran's I values:
January $2007=-0.27$, with a non-significant $z$-score of -0.49 ; February $2007=-0.08$, with a $z$-score of -0.36 ; March $2005=-0.04$, with a $z$-score of 0.62 . Thus, productive trees in a given month are randomly distributed throughout the Taï forest.

Revisiting the same trees

We followed two females for a period of 28 successive days each to specifically study what chimpanzees know during a particular season, and how they use their knowledge to navigate efficiently. We determined the revisit rate for each resource during these two periods (i.e., how frequently the resource was visited after the first known visit). The trees located less than $30 \mathrm{~m}$ from each other were considered to be the same resource. We only considered trees that were revisited by the same individual during the study period as being revisited. In order to test whether the chimpanzees revisit the same resources only by chance, we determined the revisit rate for each species studied to compare it with the simulation output.

We used a binary logistic regression to test whether social factors (party size and female ratio), density of the tree species, and tree productivity (based on the amount of time spent in the tree eating the fruit) would influence the decision to revisit a tree. To that end, we determined whether or not it was the first time a resource was being visited by creating a variable with the value of 0 if the resource was visited for the first time and was not revisited after, and a value of 1 if it was the first visit and was revisited after.

Determination of the minimum distance between trees

We created a program on visual basic in Microsoft Excel to determine whether chimpanzees went to the closest productive resource or travelled longer distances to a more 
productive resource. We only used data on the species included in the botanical map. To correct for tree productivity, we included the proportion of trees that were producing fruit during the concerned period using information extracted from the phenology data. This allowed us to create virtual maps of productive trees extracted from the complete botanical map (see above). The program used 100 different virtual maps for each species. We determined the mean distance to the next nearest resource for each of the resources considered. Then, we determined whether they walked to the next nearest resource or walked longer distances by dividing the distance effectively travelled by the distance to the next nearest resource. If the resource visited is the nearest, the variable is equal to 1 ; a value larger than 1 represents the additional distance walked from the straight line to the next nearest resource. For example, when the value equals 2 , it means that chimpanzees travelled twice the distance to the next nearest resource. We then ran a linear regression to test whether social factors (female ratio and party size), tree density, and eating duration at the tree influenced going to the next closest resource or travelling longer distances.

\section{Results}

Testing spatial memory: travelling to the closest resource

\section{Trees visited by chimpanzees}

If chimpanzees navigate without the use of spatial memory, the likelihood of finding a tree would positively correlate with species density ("no preference" simulation: $r=0.526$, $N=33, P=0.002$; " $100 \mathrm{~m}$ detection field and varying preference" simulation: $r=0.376, N=33, P=0.031$ ). However, this is not true for the "200 m varying preference" simulation, for which the simulation favours the detection of chimpanzees' rare and preferred fruit tree species ( $r=0.001, N=33, P=0.995)$. In contrast to the simulation, chimpanzees visited the least abundant tree species more frequently, as shown by the strong negative correlation with tree density $(r=-0.711, N=33, P<0.001)$ for all the concerned seasons. Therefore, the results of the simulations do not predict the frequency of chimpanzee food tree visitation. This is especially well illustrated by the very rare $\mathrm{Pou}$ teria aningueri trees that would be rarely encountered if chimpanzees were to navigate without spatial memory, but were actually the most frequently visited tree species in March 2005 (Fig. 2). The observed and simulated visit frequencies are not significantly correlated [non preference simulation (NP): Spearman Rho $r=-0.270, N=33$, $P=0.129$; varying preference $100 \mathrm{~m}$ (VP $100 \mathrm{~m}$ ): $r=-0.193, N=33, P=0.281$; varying preference $200 \mathrm{~m}$

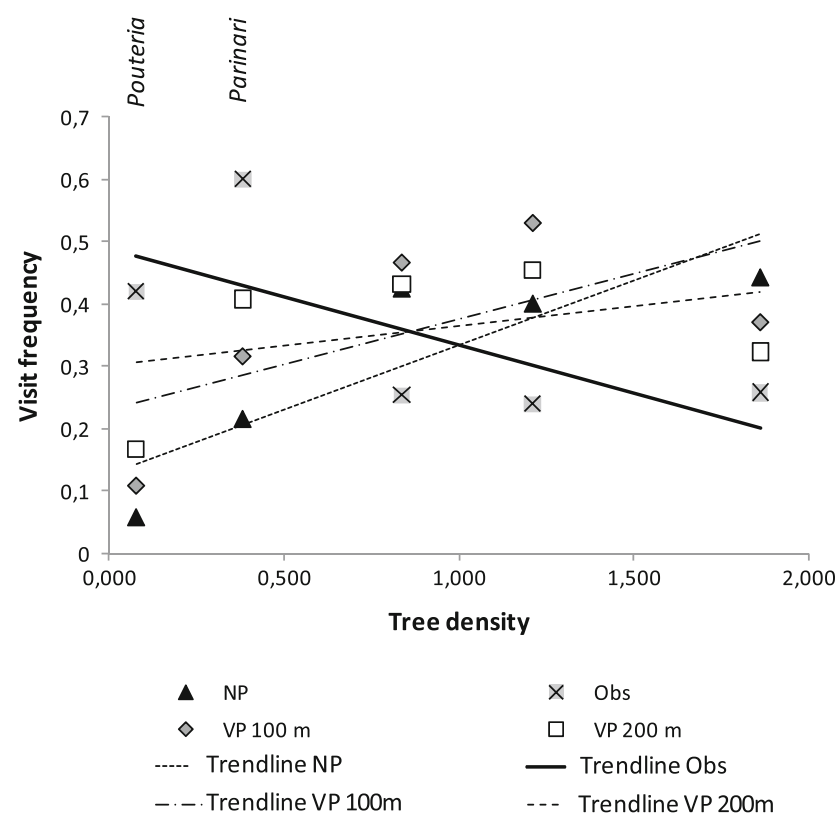

Fig. 2 Taï chimpanzees visit rare tree species more frequently: comparison between the observed and simulated frequency of visits for five different tree species with the two rare species (Pouteria aningueri and Parinari excelsa) are highlighted. The observed and simulated visit frequencies are not significantly correlated. $N P$ no preference simulation, $V P$ varying preference simulation, $O b s$ observation

(VP $200 \mathrm{~m}$ ): $r=0.176, N=33 P=0.327]$. Moreover, the observed visit frequency for all time periods is significantly higher than the different simulations for the rare tree species (Fig. 2). Thus, the "no spatial memory" model predicts the visitation frequency of abundant tree species fairly well, but not the visitation frequency of the rare species that are the trees most frequently visited by the chimpanzees (Fig. 2).

\section{Distance travelled between resources by chimpanzees}

As expected, when the detection field increases, the distance travelled to discover a resource by chance decreases (Spearman: $r=-0.394, N=96, P<0.001$ ) (see examples of a rare tree species, Parinari, and a common tree species, Xylia, in Fig. 3). In addition to this, the less strict we set the preference criteria (ranging from preference to varying to no preference), the better we were able to predict the distance travelled for the rare species. For the abundant species, the "no preference simulation" also better predicts the observed distance travelled, whereas the prediction is weaker for intermediary preference models (see Fig. 3). Overall, when comparing the simulation results for all species of trees visited, the simulations predicted a significantly longer distance than the observations in 98 out of 108 cases (this number comes from one or three different tree species visited by the chimpanzees during four different months' periods, representing nine conditions for which we did the 12 different simulations seen in Fig. 3). 


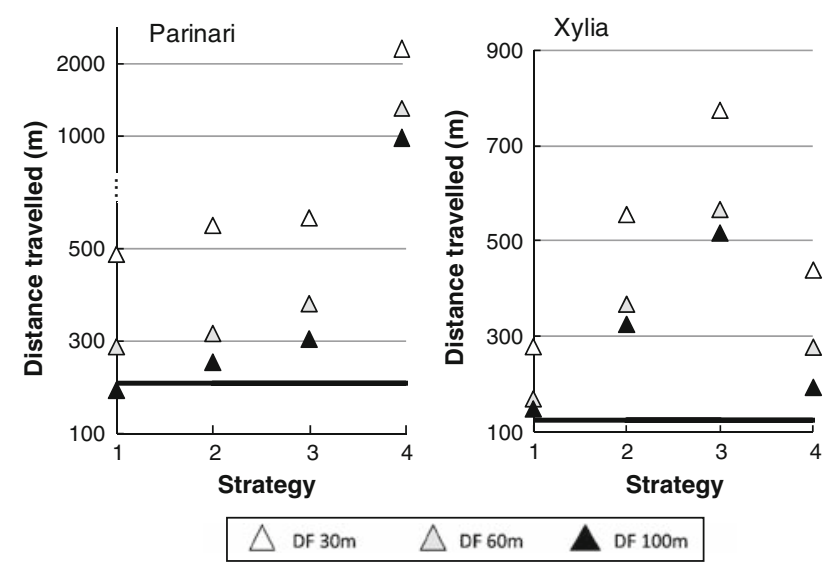

Fig. 3 Taï chimpanzees travelled shorter distances than expected without spatial memory. The figure presents the results of all simulations for Parinari excelsa, a rare tree species (a), and the Xylia evansii, an abundant tree species (b) for January. The bold dark horizontal line represents the observed distance travelled. The three detection field widths are simulated ( $D F 30 \mathrm{~m}, D F 60 \mathrm{~m}$ and $D F 100 \mathrm{~m}$ ) as well as four alternative preference models ( 1 non preference simulation, 2 varying preference after $100 \mathrm{~m}, 3$ varying preference after $200 \mathrm{~m}$, 4 preference simulation). a All simulations for Parinari significantly predict longer distances than the observations, except for the non-preference simulation with a 100-m detection field (black triangle). b Here, all simulations for Xylia predict longer distances than the observations

The poor predictive values of the simulations without mental maps is further stressed by the fact that, in the very rare case where the distance travelled is well predicted, that is with a high detection field of $100 \mathrm{~m}$ and no preference for rare tree species, like Parinari $(t=-1.478, d f=99$, $P=0.143$ ), the frequency of visits is dramatically underestimated (see Figs. 2, 3). Similarly, when the frequency of visits is correctly predicted for abundant trees, like Xylia, all simulations predict longer distances than what was actually observed $(Z=-3.181, N=15, P=0.001)$ (see Figs. 2, 3). However, it remains that the deviation between observations and all simulations is smaller for abundant tree species than for rare ones.

\section{Travelling to the next closest resource}

The previous analysis showed that tree visits by chimpanzees could not be explained in the Taï forest without the use of a spatial memory. Now the question remains as to what criteria chimpanzees use when selecting a particular tree. We first tested whether chimpanzees simply travel to the next closest resources. Our results indicate that chimpanzees go to the next closest productive resource in $30 \%$ of the cases. Alternatively, social factors, as well as tree species density and productivity, or time spent eating in a tree could influence the travel distance. Linear regression analyses show that the more productive the target trees are and the longer they spent eating in the tree, the longer the distances the chimpanzees travel $(B=0.248, t=2.465, d f=1$, $P=0.014$ ) (Fig. 4a). This effect is amplified when the party is composed of more males than females $(B=-1.401$, $t=1.401, d f=1, P<0.001$ ) (Fig. 4a). However, party size has no effect on distance travelled $(B=-0.015, t=0.455$, $P=0.650$ ). In addition, for rare tree species, chimpanzees tended to walk to the next closest resource, while for more abundant tree species they travelled longer distances ( $B=0.464, t=0.464, d f=1, P<0.001)$ to select the more productive trees where they could spend a longer time feeding (see Fig. 4b). This is logical in terms of minimizing travel cost as travelling longer distances than the next closest tree for rare food resources would be very energy consuming, while travelling longer distances for abundant resources would not.
Fig. 4 a Chimpanzees travel further to reach trees with more fruit. This effect is stronger for males (triangles) in groups or alone than for females (squares). b Chimpanzees travel further to reach trees of the more abundant species
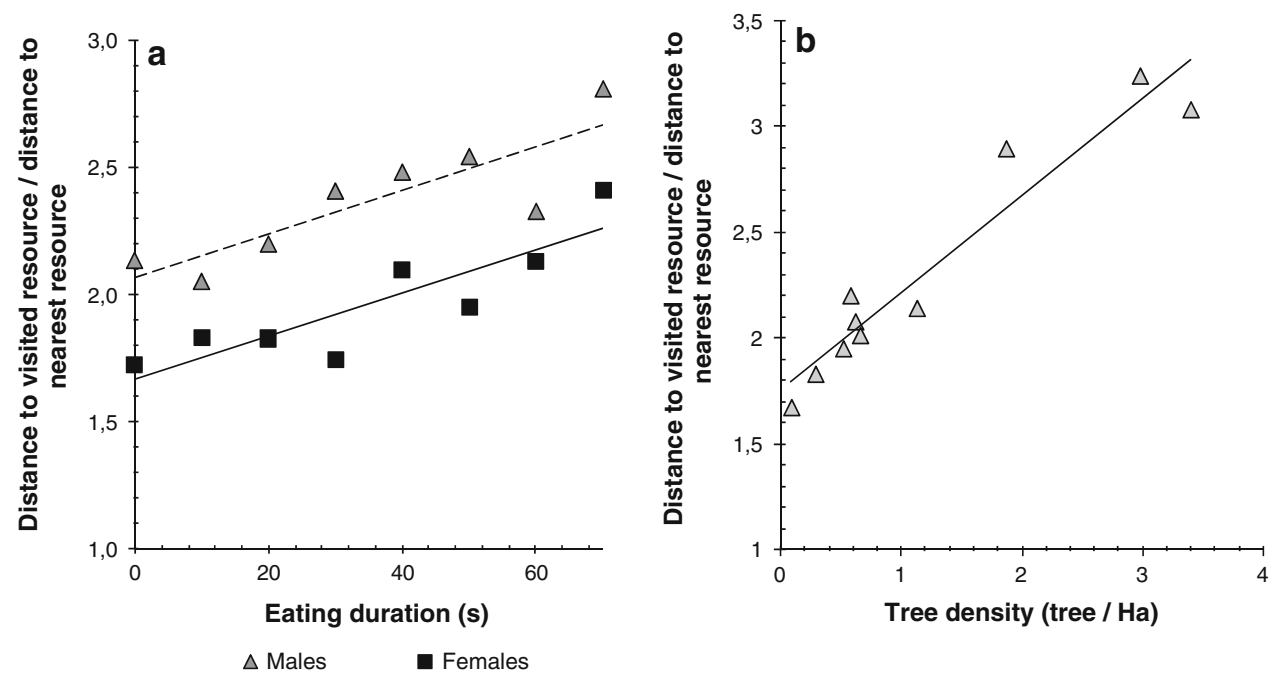
Fig. 5 Chimpanzees revisit resources more often than expected by chance: testing for revisit rate without spatial memory, when chimpanzees have a preference for one tree species as they travel. Points are on the bold continuous line when the simulation results equal the observations, but are on the right side of the line when simulation results predict a lower revisit rate than observations

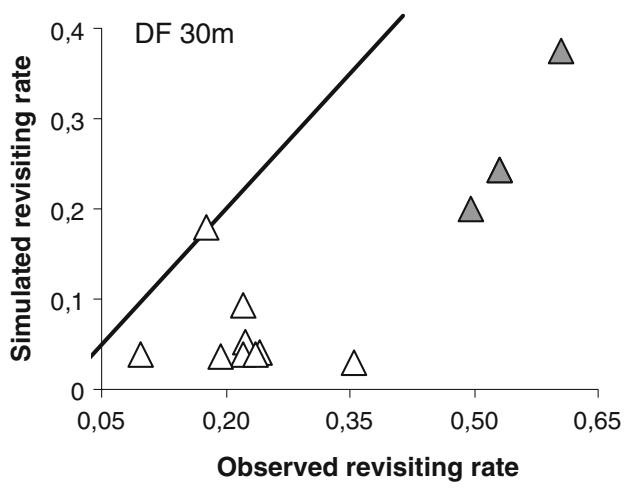

$\triangle$ Abundant tree species

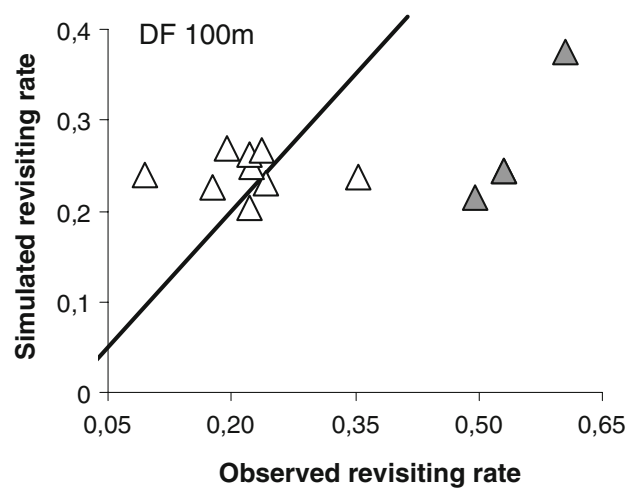

$\triangle$ Rare Species $\triangle$ Abundant tree species
Testing spatial memory: criteria for revisit

\section{Chimpanzees revisit the same trees}

On average, chimpanzees revisit a tree within 5.37 days. However, due to the fact that we never followed the same target for more than 28 consecutive days, the longest interval between revisits was 24 days. Of the two females that we followed over long periods of time, one ate in 391 different trees, with an average of 13.96 trees per day, and the second ate in 506 trees, with an average of 18.07 trees per day. Without spatial memory and preference for a tree species, the revisit rate from the simulation with a detection field of $30 \mathrm{~m}$ (Wilcoxon $N=13, Z=-3.11 P=0.002$ ) and $60 \mathrm{~m}$ (Wilcoxon $N=13, Z=-2.97 P=0.003$ ) is significantly lower than the one observed in the chimpanzees. However, the simulation with a detection field of $100 \mathrm{~m}$ and observed revisit rates are similar (Wilcoxon $N=13$, $Z=-0.8 P=0.422$ ). When considering these results in detail, simulated revisit rates for rare species are significantly different from what we observed (Fig. 5), while simulated revisit rates for abundant species are similar to observed revisit rates when the detection field is $100 \mathrm{~m}$ (Fig. 5, DF 100). Consequently, chimpanzees would not revisit rare species as often as we observed them to if they were navigating without spatial memory.

To understand why chimpanzees revisit some individual trees and not others, we ran a binary logistic regression to test the effects of social factors (party size, female ratio), tree species density, and duration of time spent eating in the tree. The probability of revisiting a tree is strongly and positively affected by the amount of time spent eating in that particular tree during the previous visit $(B=0.506$ Wald $=22.252, d f=1 P<0.001)$, and the female ratio $(B=1.438$ Wald $=13.412, d f=1, P<0.001)$ (Fig. 6), and is negatively affected by the tree species density $(B=-0.337$, Wald $=19.420, d f=1, P<0.001)$, as seen previously. However, there is no influence of party size on the decision to revisit a tree $(B=-0.029$, Wald $=0.762$, $d f=1, P=0.383)$.

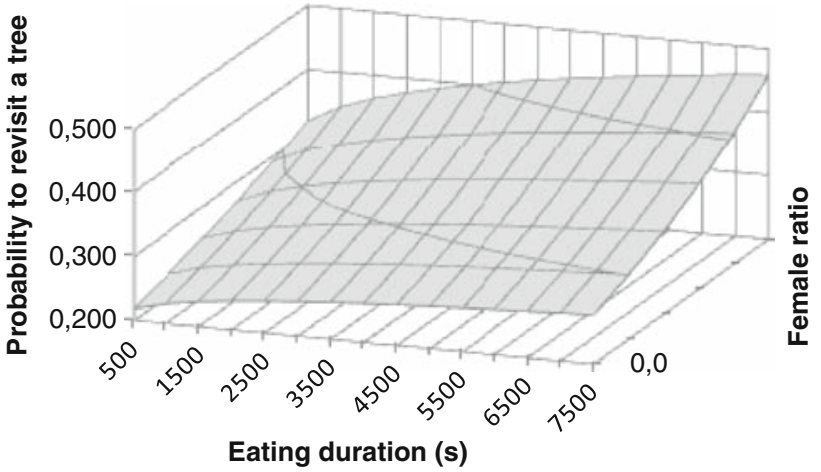

Fig. 6 Taï chimpanzees revisit trees in which they ate for longer periods of time and when there was a higher proportion of females in the party in the previous visit significantly more frequently. The $3 \mathrm{D}$ figure represents the statistical model determined by the binary logistic regression

\section{Discussion}

The primary result from our analysis is that Taï chimpanzees are able to efficiently reach one tree out of the thousands available at any given time in their territory. The rare tree species, like the Parinari excelsa with a density of 0.382 trees per hectare, are detected by chance too rarely to explain the observations. This means that, for all the rare tree species, chimpanzees must know the precise location of these trees. For the abundant species, the simulation results still fail to predict the exact distance needed to reach them, as seen from the observations of the chimpanzees. Does that mean that chimpanzees could still find the trees without spatial memory? We believe that the fact that the simulation results were closer to the observations for abundant trees might indicate that another strategy could have been used without remembering the location of all individual trees. Moreover, it could still be possible for chimpanzees to only remember the location of the few clusters of abundant tree species in the territory and look at random within the clusters for individual trees. This could be the case for one large Xylia tree cluster found in South Group 
territory (see Fig. 1). However, out of the 17 tree species mapped, 11 have a density lower than 0.5 trees per hectare and our simulations show that chimpanzees need to remember them individually to be able to find them as often as they do and with such efficient distance travelled. In addition, the chimpanzees not only remember the trees' locations but are also able to associate information about previous feeding times in the particular trees.

The question remains as to whether captive ape studies can adequately capture the breadth of the spatial abilities required by wild animals in their natural environment. Unfortunately, due to the limitations inherently introduced under the captive conditions, animals were only tested with a limited number of objects presented in a limited space. Our results suggest that the spatial memory in Taï chimpanzees reaches a dimension that is many times greater than what could be demonstrated with captive individuals (Menzel 1973). It is difficult to know if captive individuals are impaired in their spatial memory, as such abilities are never required, or if experiments carried out in captive settings were not demanding enough to reflect their real capabilities. Some evidence of impairments in captive individuals' versus wild individuals' spatial knowledge suggests that the former explanation might be more correct (Menzel and Beck 2000). It is important to note here that the challenge in the spatial domain is much more important in wild than in captive conditions, and individuals living in the wild face such challenges starting at a very early age. Therefore, it should come as no surprise that they would demonstrate higher spatial abilities. Previous analyses have convincingly shown that cognitive abilities develop only when they are required (Tang et al. 2006; Boesch 2007; Nelson et al. 2007; Beller and Bender 2008).

Spatial memory performance in Taï chimpanzees seems to be adapted to the ecological properties of the tree species. Distance travelled is more carefully selected for rare trees whereas for abundant tree species, chimpanzees seem to remember and select richer and more productive fruiting trees and give the distance needed to travel to these resources much less consideration. These are the optimal solutions in both cases and suggest a high level of flexibility in the way that chimpanzees use their spatial memory.

Available resources are abundant during some seasons and navigating randomly in any direction could eventually lead to a productive tree. However, this would not be an efficient means of navigation since low-density tree species would be infrequently encountered and would lead individuals to travel many kilometres. Chimpanzees navigate efficiently through abundant resources and revisit the same resource more often than expected by chance. They travel efficiently, by minimizing the distance they travel. However, they only travel to the next closest resource on rare occasions. This positive interaction between distance travelled and eating duration means that, for a set of available resources, chimpanzees bypass less productive resources to select more productive ones, as has been previously shown in Saki monkeys (Cunningham and Janson 2007). Moreover, they revisit the resource where they are able to eat for longer and where they expect to find more fruit. Therefore, chimpanzees have a powerful spatial memory that allows them to select the most attractive resource. To be able to select more productive resources over others, chimpanzees need to remember the location of a very large number of resources. In addition, to have a more precise idea of what they know about their environment and how many resources they are able to remember, it would be necessary to know whether the time spent eating at a tree is proportional to the fruit abundance of the tree, as well as to evaluate the abundance of fruit where they eat and the trees of the same species that were closer than the one visited.

Sex differences have been observed in Taï chimpanzees, with females travelling shorter distances and in a more linear way than males (Normand and Boesch 2009). Further, we have shown that female ratio influences revisiting trees and the distance travelled to resources. In addition, a party with more females has been shown to travel shorter distances than a party with more males. The tendency of females to select the closest resource is not a consequence of party size, meaning that it is not a response to possible feeding competition. Such sex differences seem to be compatible with an evolutionary scenario proposing that males compete with each other through extensive ranging, meaning that they would explore their territory more and have a larger overview (Ecuyer-Dab and Robert 2004), while, in contrast, females would develop sex-specific gathering activities that would strengthen differential spatial cognitive abilities (Gaulin and FitzGerald 1986) and, therefore, a superior spatial memory than males.

However, further analyses are necessary to better understand the origin of these sex differences as our present study and findings are not able to provide insight into them. First, this is because our results do not allow us to determine whether females have a more precise spatial memory that allows them to select closer productive resources, or whether males know more about the fruiting state and navigate to more productive resources than females. Second, hormonal influences should also be tested as they have been shown to influence female performance on spatial tasks in both humans (Postma et al. 1999), and primates (Lacreuse et al. 2001). These sex differences are interesting because precise spatial memory in females (including their advanced skills in Panda nut cracking, and locating hammers, Boesch and Boesch-Achermann 2000) is a necessity, considering that, due to pregnancies and suckling, they are more dependent on high food quality than males, who range more widely. Thus, it would be interesting to see if 
the differences observed in chimpanzees are due to different strategies or different skills. Can females travel to closer resources than males because they know more resources, or is it because males are avoiding competition so that they select more productive resources? This could also be tackled with more detailed information about tree productivity by testing whether females visit less productive resources than males.

Overall, our results show that it is possible to study spatial cognition in wild animals, as long as exact data on all possible food sources are obtained (Janson and Byrne 2007). Simulations proved to be a very powerful tool to control and exclude many possible alternative strategies that cannot be directly tested with wild animals (Janson 1998; Garber and Hannon 1993). The main advantage of such an approach is that it allows us to decipher the spatial abilities and memory capacities that are required to solve the real challenges faced daily by animals in rich and complex forest environments (Boesch 1993; Gould 2004). This study represents a first step in understanding more about spatial memory in wild chimpanzees and reveals that the spatial memory required in the wild is extensive. Further, this paper raises interesting questions about spatial memory capacities and sex differences. It also shows that, with precise knowledge about the availability of the resources, it is possible to understand exactly how chimpanzees' cognitive abilities can be adapted to highly challenging situations.

Acknowledgments We thank the 'Ministère de la Recherche Scientifique' and the 'Ministère des Eaux et Forêts' of Côte d'Ivoire, the director of the OIPR and the director of the Taï National Park for permission to conduct this study. We also thank the 'Centre Suisse de la Recherche Scientifique' in Abidjan, the 'Centre de Recherche en Écologie Tropicale', the 'Projet Singe' and especially the 'Projet Chimpanzé' in Taï for their help. We are really grateful to Michel Langlais for his constant support and advice. Assistance in data collection was provided by Nicaise Oulaï, Nestor Gouyan Bah, Jonas Tahou, François Yro and Jean-Baptiste Mihi Nahan. We thank Dervla Dowd and Carolyn Rowney for improving the English of this manuscript. This research was funded by the Max Planck Society, and the French Ministry of Research.

Open Access This article is distributed under the terms of the Creative Commons Attribution Noncommercial License which permits any noncommercial use, distribution, and reproduction in any medium, provided the original author(s) and source are credited.

\section{References}

Beatty WW, Shavalia DA (1980) Spatial memory in rats: time course of working memory and effect of anesthetics. Behav Neurol Biol 28:454-462

Beller S, Bender A (2008) The limits of counting: numerical cognition between evolution and culture. Science 319:213-215. doi:10.1126/science. 1148345

Boesch C (1993) Toward a new image of culture in chimpanzees. Behav Brain Sci 16:514-515
Boesch C (2007) What makes us human (Homo sapiens)? The challenge of cognitive cross-species comparison. J Comp Psychol 121(3):227-240. doi:10.1037/0735-7036.121.3.227

Boesch C, Boesch-Achermann H (2000) The chimpanzees of the Tai Forest: behavioural ecology and evolution. Oxford University Press, Oxford

Boesch C, Goné Bi ZB, Anderson D, Stahl D (2006a) Food choice in Taï chimpanzees: are cultural differences present? In: Hohmann G, Robbins MM, Boesch C (Eds) Feeding ecology in Apes and other primates. Cambridge studies in Biological and Evolutionary Anthropology, pp. 183-201

Boesch C, Kohou G, Néné H, Vigilant L (2006b) Male competition and paternity in wild chimpanzees of the Taï forest. Am J Phys Anthropol 130:103-115

Boesch C, Crockford C, Herbinger I, Wittig R, Moebius Y, Normand E (2008) Intergroup conflicts among chimpanzees in Taï National Park: lethal violence and the female perspective. Am J Primatol 70:1-14

Clayton NS, Dickinson A (1998) Episodic-like memory during cache recovery by scrub jays. Nature 395:272-274

Cunningham EP, Janson CH (2007) Integrating information about location and value of resources by white faced-saki monkeys (Pithecia pithecia). Anim Cogn 10:293-304. doi:10.1007/ s10071-007-0077-4

Dumont B, Petit M (1998) Spatial memory of sheep at pasture. Appl Anim Behav Sci 60:43-53

Ecuyer-Dab I, Robert M (2004) Have sex differences in spatial ability evolved from male competition for mating and female concern for survival? Cognition 91:221-257. doi:10.1016/j.cognition.2003. 09.007

Garber PA (1988) Foraging decisions during nectar-feeding by tamarin monkey (Saguinus mystax and Saguinus fuscicollis). Biotropica 20:100-106

Garber PA (1989) Role of spatial memory in primate foraging patterns in Saguinus mystax and Saguinus fuscicollis. Am J Primatol 19:203-216

Garber PA, Hannon B (1993) Modeling monkeys: a comparison of computer-generated and naturally occurring foraging patterns in two species of neotropical primates. Int J Primatol 14:827-852

Garber PA, Paciulli LM (1997) Experimental field study of spatial memory and learning in wild capuchin monkeys (Cebus capucinus). Folia Primatol 68:236-253

Gaulin SJC, FitzGerald RW (1986) Sex differences in spatial ability: an evolutionary hypothesis and test. Am Nat 127:74-88

Gould JL (2004) Animal cognition. Curr Biol 14:372-375

Held S, Baumgartner J, KilBride A, Byrne RW, Mendl M (2005) Foraging behaviour in domestic pigs (Sus scrofa): remembering and prioritizing food sites of different value. Anim Cogn 8:114-121. doi:10.1007/s10071-004-0242-y

Janmaat KRL, Byrne RW, Zuberbühler K (2006) Evidence for a spatial memory of fruiting states of rainforest trees in wild mangabeys. Anim Behav 72:797-807. doi:10.1016/j.anbehav.2005.12.009

Janson CH (1998) Experimental evidence for spatial memory in foraging wild capuchin monkeys Cebus apella. Anim Behav 55:12291243

Janson CH (2007) Experimental evidence for route integration and strategic planning. Anim Cogn 10:341-356. doi:10.1007/s10071007-0079-2

Janson CH, Byrne RW (2007) What wild primates know about resources: opening up the black box. Anim Cogn 10:357-367. doi:10.1007/s10071-007-0080-9

Lacreuse A, Verreault M, Herndon JG (2001) Fluctuations in spatial recognition memory across the menstrual cycle in female rhesus monkeys. Psychoneuroendocrinology 26:623-639

Menzel EW (1973) Chimpanzee spatial memory organization. Science 182:943-945 
Menzel CR, Beck BB (2000) Homing and detour behavior in golden lion tamarin social groups. In: Boinski S, Garber P (Eds) On the move; how and why animals travel in groups. Chicago University of Chicago Press, Chicago, pp. 299-326

Morris RGM (1981) Spatial localization does not require the presence of local cues. Learn Motiv 12:239-260

Nelson C, Zeanah C, Fox N, Marshall P, Smyke A, Guthrie D (2007) Cognitive recovery in socially deprived young children: the Bucharest early intervention project. Science 318:1937-1940. doi:10.1126/science. 1143921

Normand E, Boesch C (2009) Sophisticated mental maps in forest chimpanzees. Anim Behav 77:1195-1201
Noser R, Byrne RW (2007) Travel routes and planning of visits to outof-sight resources in wild chacma baboons Papio ursinus. Anim Behav 10:331-341. doi:10.1016/j.anbehav.2006.04.012

Postma A, Winkel J, Tuiten A, Van Honk J (1999) Sex differences and menstrual cycle effects in human spatial memory. Psychoneuroendocrinology 24:175-192

Raby CR, Alexis DM, Dickinson A, Clayton NS (2007) Planning for the future by Western Scrub-Jays. Nature 445:919-921. doi:10.1038/nature05575

Tang Y, Zhang W, Chen K, Feng S, Ji Y, Shen J, Reiman E, Liu Y (2006) Arithmetic processing in the brain shaped by cultures. Proc Natl Acad Sci 103:10775-10780 